\title{
On a weak Gauß law in general relativity and torsion
}

\author{
Thomas Schücker1 $\left(\mathrm{CPT}^{2}\right)$, Sami R. ZouZou年 (LPT包)
}

\begin{abstract}
We present an explicit example showing that the weak Gauß law of general relativity (with cosmological constant) fails in Einstein-Cartan's theory. We take this as an indication that torsion might replace dark matter.
\end{abstract}

PACS: 04.20.-q

Key-Words: Einstein equation, general relativity

CPT-P005-2012

1203.5642

\footnotetext{
${ }^{1}$ thomas.schucker@gmail.com

${ }^{2}$ Centre de Physique Théorique, Aix-Marseille Univ; CNRS, UMR 7332; Univ Sud Toulon Var; 13288 Marseille Cedex 9, France

${ }^{3}$ szouzou2000@yahoo.fr

${ }^{4}$ Laboratoire de Physique Théorique, Département de Physique, Faculté des Sciences Exactes, Univ Mentouri-Constantine, Algeria
} 


\section{Introduction}

Gauß' law in Maxwell's theory allows to link the total charge of an isolated charge distribution to the asymptotic strength of its electric field. In general relativity a weak form of Gauß' law holds in the static and spherically symmetric case and remains true when a cosmological constant is added. We show that this weak Gauß law fails when torsion is added à la Einstein-Cartan [1, 2, 3].

\section{The weak Gauß law of general relativity}

To warm up, let us quickly review the derivation of the weak Gauß law in general relativity with cosmological constant.

In a first step we solve the Killing equation

$$
\xi^{\alpha} \frac{\partial}{\partial x^{\alpha}} g_{\mu \nu}+\frac{\partial \xi^{\bar{\mu}}}{\partial x^{\mu}} g_{\bar{\mu} \nu}+\frac{\partial \xi^{\bar{\nu}}}{\partial x^{\nu}} g_{\mu \bar{\nu}}=0 .
$$

in the static, spherical case, i.e. for the four vector fields: $\xi=\partial / \partial t$ generating time translation and

$$
\begin{aligned}
\xi & =-\sin \varphi \frac{\partial}{\partial \theta}-\cos \varphi \frac{\cos \theta}{\sin \theta} \frac{\partial}{\partial \varphi} \\
\xi & =+\cos \varphi \frac{\partial}{\partial \theta}-\sin \varphi \frac{\cos \theta}{\sin \theta} \frac{\partial}{\partial \varphi} \\
\xi & =\frac{\partial}{\partial \varphi}
\end{aligned}
$$

generating the rotations around the $x$-, $y$ - and $z$-axis. In fact, since for the infinitesimal rotations, the commutator of the last two gives the first, we may discard the infinitesimal rotation around the $x$-axis. It is a typical accountant's work to list and solve the remaining $3 \times 10$ linear, first order partial differential equations for the 10 unknowns $g_{\mu \nu}$. The solution is well known:

$$
\mathrm{d} \tau^{2}=B \mathrm{~d} t^{2}+2 D \mathrm{~d} t \mathrm{~d} r-A \mathrm{~d} r^{2}-C \mathrm{~d} \theta^{2}-C \sin ^{2} \theta \mathrm{d} \varphi^{2}
$$

with four functions $A, B, C$ and $D$ of $r$. By a suitable coordinate transformation we may achieve $D=0$ and $C=r^{2}$. Then $A$ and $B$ are positive.

In a second step we solve the Einstein equation

$$
\text { Ricci }_{\mu \nu}-\frac{1}{2} \text { scalar } g_{\mu \nu}-\Lambda g_{\mu \nu}=8 \pi G \tau_{\mu \nu}
$$

in the vacuum, $\tau_{\mu \nu}=0$, and obtain the Kottler or Schwarzschild - de Sitter solution:

$$
B=\frac{1}{A}=1-\frac{S}{r}-\frac{1}{3} \Lambda r^{2}
$$

with an integration constant $S$. 
In a third step we use the weak Gauß law to determine the integration constant $S$ in terms of the total mass of a spherically symmetric mass distribution $\tau_{t}^{t}(r)$ with support inside a ball of radius $R$ (not to be confused with the curvature scalar, that we denote by 'scalar'). This law is simply the ${ }_{t}^{t}$ component of the Einstein equation:

$$
\operatorname{Ricci}_{t}^{t}-\frac{1}{2} \text { scalar } g_{t}^{t}-\Lambda g_{t}^{t}=8 \pi G \tau^{t}{ }_{t} .
$$

In the static, spherical case this equation reduces to:

$$
\frac{1}{r^{2}}\left[1-\frac{\mathrm{d}}{\mathrm{d} r} \frac{r}{A}\right]-\Lambda=8 \pi G \tau_{t}^{t}
$$

Solving for $\mathrm{d}(r / A) / \mathrm{d} r$ and integrating we have:

$$
\frac{r}{A}=r-2 G \int_{0}^{r} \tau_{t}^{t}(\tilde{r}) 4 \pi \tilde{r}^{2} \mathrm{~d} \tilde{r}-\frac{1}{3} \Lambda r^{3}+K .
$$

The integration constant $K$ is seen to vanish by evaluating at $r=0$ and noting that $A(0)$ is positive. Then the interior solution is

$$
A(r)=\left[1-\left(2 G \int_{0}^{r} \tau_{t}^{t}(\tilde{r}) 4 \pi \tilde{r}^{2} \mathrm{~d} \tilde{r}\right) / r-\frac{1}{3} \Lambda r^{2}\right]^{-1}
$$

and from the continuity of $A$ at $r=R$ we obtain the Schwarzschild radius $S=2 G M$ with

$$
M:=\int_{0}^{R} \tau_{t}^{t}(\tilde{r}) 4 \pi \tilde{r}^{2} \mathrm{~d} \tilde{r} .
$$

\section{The weak Gauß law in Einstein-Cartan's theory}

Our task is to redo the above three steps including torsion à la Einstein-Cartan.

\subsection{First step: invariant connection}

We have to solve the analogue of the Killing equation [4] for the now independent (metric) connection $\Gamma$

$$
\xi^{\alpha} \frac{\partial}{\partial x^{\alpha}} \Gamma_{\mu \nu}^{\lambda}-\frac{\partial \xi^{\lambda}}{\partial x^{\bar{\lambda}}} \Gamma_{\mu \nu}^{\bar{\lambda}}+\frac{\partial \xi^{\bar{\mu}}}{\partial x^{\mu}} \Gamma_{\bar{\mu} \nu}^{\lambda}+\frac{\partial \xi^{\bar{\nu}}}{\partial x^{\nu}} \Gamma_{\mu \bar{\nu}}^{\lambda}+\frac{\partial^{2} \xi^{\lambda}}{\partial x^{\mu} \partial x^{\nu}}=0
$$

for the vector fields generating time translation and rotations. These $3 \times 64$ equations yield the following non-vanishing connection components:

$$
\begin{aligned}
\Gamma_{b c}^{a}=X_{b c}^{a}(r), & a, b, c \in\{t, r\}, \\
\Gamma_{\theta \theta}^{a}=\Gamma_{\varphi \varphi}^{a} / \sin ^{2} \theta=E^{a}(r), & \Gamma_{\theta \varphi}^{a}=-\Gamma^{a}{ }_{\varphi \theta}=\sin \theta F^{a}(r), \\
\Gamma_{a \theta}^{\theta}=\Gamma_{a \varphi}^{\varphi}=C_{a}(r), & \Gamma_{\theta a}^{\theta}=\Gamma_{\varphi a}^{\varphi}=Y_{a}(r), \\
\Gamma_{\varphi \varphi}^{\theta}=-\sin \theta \cos \theta, & \Gamma^{\varphi}{ }_{\theta \varphi}=\Gamma_{\varphi \theta}^{\varphi}=\cos \theta / \sin \theta,
\end{aligned}
$$


with $8+2+2+2+2=16$ arbitrary functions $X, E, F, C, Y$ of $r$. The metricity condition,

$$
\frac{\partial}{\partial x^{\lambda}} g_{\mu \nu}-\Gamma_{\mu \lambda}^{\bar{\mu}} g_{\bar{\mu} \nu}-\Gamma_{\nu \lambda}^{\bar{\nu}} g_{\mu \bar{\nu}}=0
$$

reduces the 16 functions to four arbitrary functions $C_{t}, C_{r}, D_{t}, D_{r}$ and we have the following non-vanishing connection components:

$$
\begin{aligned}
\Gamma_{t r}^{t}=\frac{1}{2} B^{\prime} / B, \quad \Gamma_{r a}^{t}=D_{a} A / B, & \Gamma_{r r}^{r}=\frac{1}{2} A^{\prime} / A, \quad \Gamma_{t a}^{r}=D_{a}, \\
\Gamma_{\theta \theta}^{t}=\Gamma_{\varphi \varphi}^{t} / \sin ^{2} \theta=C_{t} r^{2} / B, & \Gamma_{\theta \theta}^{r}=\Gamma_{\varphi \varphi}^{r} / \sin ^{2} \theta=-C_{r} r^{2} / A, \\
\Gamma_{a \theta}^{\theta}=\Gamma_{a \varphi}^{\varphi}=C_{a}, & \Gamma_{\theta r}^{\theta}=\Gamma_{\varphi r}^{\varphi}=1 / r \\
\Gamma_{\varphi \varphi}^{\theta}=-\sin \theta \cos \theta, & \Gamma_{\theta \varphi}^{\varphi}=\Gamma_{\varphi \theta}^{\varphi}=\cos \theta / \sin \theta .
\end{aligned}
$$

\subsection{Einstein's equation}

So far we have used a holonomic frame $\mathrm{d} x^{\mu}$. Now it will be convenient to work in an orthornormal frame $e^{a}=: e^{a}{ }_{\mu} \mathrm{d} x^{\mu}$. We will use the notations of reference [5]. The metric tensor reads $g_{\mu \nu}(x)=e^{a}{ }_{\mu}(x) e^{b}{ }_{\nu}(x) \eta_{a b}$.

The connection with respect to a holonomic frame is written as a $g \ell(4)$-valued 1 -form $\Gamma_{\beta}^{\alpha}=: \Gamma_{\beta \mu}^{\alpha} \mathrm{d} x^{\mu}$. The link between the components of the same connection with respect to the holonomic frame $\Gamma$ and with respect to the orthonormal frame $\omega$ is given by the $G L(4)$ gauge transformation with $e(x)=e^{a}{ }_{\mu}(x) \in G L(4)$;

$$
\omega=e \Gamma e^{-1}+e \mathrm{~d} e^{-1}
$$

or with indices:

$$
\omega_{b \mu}^{a}=e_{\alpha}^{a} \Gamma_{\beta \mu}^{\alpha} e_{b}^{-1 \beta}+e_{\alpha}^{a} \frac{\partial}{\partial x^{\mu}} e^{-1 \alpha}{ }_{b}
$$

For $\omega$ the metricity condition is algebraic and means that its values $\omega_{b}^{a}$ are in the Lie algebra of the Lorentz group: $\omega_{a b}=-\omega_{b a}$.

In the orthonormal frame with $e=\operatorname{diag}(\sqrt{B}, \sqrt{A}, r, r \sin \theta)$, the non-vanishing components $\omega_{b \mu}^{a}$ of the invariant connection (19-22) are:

$$
\begin{aligned}
\omega_{r t}^{t}=D_{t} \sqrt{A / B}, \quad \omega_{r r}^{t} & =D_{r} \sqrt{A / B}, \quad \omega_{\theta \theta}^{t}=\omega_{\varphi \varphi}^{t} / \sin \theta=C_{t} r / \sqrt{B}, \\
\omega_{\varphi \varphi}^{\theta} & =-\cos \theta, \quad \omega_{\theta \theta}^{r}=\omega_{\varphi \varphi}^{r} / \sin \theta=-C_{r} r / \sqrt{A} .
\end{aligned}
$$

The curvature as $s o(1,3)$-valued 2-form,

$$
R:=\mathrm{d} \omega+\frac{1}{2}[\omega, \omega]
$$

has the following non-vanishing components, $R_{b}^{a}=: \frac{1}{2} R_{b \mu \nu}^{a} \mathrm{~d} x^{\mu} \mathrm{d} x^{\nu}$ :

$$
\begin{aligned}
& R_{r t r}^{t}=-\left(D_{t} \sqrt{A / B}\right)^{\prime}, \quad R_{\theta t \theta}^{t}=-C_{r} D_{t} r / \sqrt{B}, \quad R_{\theta r \theta}^{t}=\left(C_{t} r / \sqrt{B}\right)^{\prime}-C_{r} D_{r} r / \sqrt{B}, \\
& R_{\varphi t \varphi}^{t}=\sin \theta R_{\theta t \theta}^{t}, \quad R_{\varphi r \varphi}^{t}=\sin \theta R_{\theta r \theta}^{t}, \\
& R_{\theta t \theta}^{r}=C_{t} D_{t} r \sqrt{A} / B, \quad R_{\theta r \theta}^{r}=-\left(C_{r} r / \sqrt{A}\right)^{\prime}+C_{t} D_{r} r \sqrt{A} / B \text {, } \\
& R_{\varphi t \varphi}^{r}=\sin \theta R_{\theta t \theta}^{r}, \quad R_{\varphi r \varphi}^{r}=\sin \theta R_{\theta r \theta}^{r}, \\
& R_{\varphi \theta \varphi}^{\theta}=\sin \theta\left(1+C_{t}^{2} r^{2} / B-C_{r}^{2} r^{2} / A\right) \text {. }
\end{aligned}
$$


Next we compute the Ricci tensor,

$$
\operatorname{Ricci}_{b}^{a}:=\eta^{a a^{\prime}} R_{a^{\prime} \mu \nu}^{c} e^{-1 \mu}{ }_{c} e^{-1 \nu}{ }_{b},
$$

whose non-vanishing components are:

$$
\begin{aligned}
\operatorname{Ricci}^{t} & =\left(D_{t} \sqrt{A / B}\right)^{\prime} / \sqrt{A B}+2 C_{r} D_{t} / B \\
\operatorname{Ricci}^{r}{ }_{r} & =\left(D_{t} \sqrt{A / B}\right)^{\prime} / \sqrt{A B}+2\left(r C_{r} / \sqrt{A}\right)^{\prime} /(r \sqrt{A})-2 C_{t} D_{r} / B \\
\operatorname{Ricci}_{r}{ }_{r} & =-2\left(C_{t} / \sqrt{B}\right)^{\prime} / \sqrt{A}-2 C_{t} /(r \sqrt{A B})+2 C_{r} D_{r} / \sqrt{A B} \\
\operatorname{Ricci}^{r} & =-2 C_{t} D_{t} \sqrt{A / B} / B \\
\operatorname{Ricci}^{\theta}{ }_{\theta} & =\operatorname{Ricci}_{\varphi}^{\varphi} \\
& =\left(C_{r} / \sqrt{A}\right)^{\prime} / \sqrt{A}-1 / r^{2}+C_{r} /(r A)-C_{t}^{2} / B+C_{r}^{2} / A-C_{t} D_{r} / B+C_{r} D_{t} / B
\end{aligned}
$$

The curvature scalar is

$$
\begin{aligned}
\text { scalar }= & \operatorname{Ricci}^{t}+\operatorname{Ricci}_{r}{ }_{r}+2 \operatorname{Ricci}^{\theta}{ }_{\theta} \\
= & 2\left(D_{t} \sqrt{A / B}\right)^{\prime} / \sqrt{A B}+4\left(C_{r} / \sqrt{A}\right)^{\prime} / \sqrt{A}-2 / r^{2}+4 C_{r} /(r A) \\
& -2 C_{t}^{2} / B+2 C_{r}^{2} / A-4 C_{t} D_{r} / B+4 C_{r} D_{t} / B .
\end{aligned}
$$

In an orthonormal frame the Einstein equation reads

$$
\operatorname{Ricci}_{b}{ }_{b}-\frac{1}{2} \operatorname{scalar} \delta^{a}{ }_{b}-\Lambda \delta^{a}{ }_{b}=8 \pi G \tau_{b}{ }^{a} .
$$

In our orthonormal frame $e=\operatorname{diag}(\sqrt{B}, \sqrt{A}, r, r \sin \theta)$, the energy momentum tensor $\tau_{a b}$ reads

$$
\tau_{b}^{a}=\left(\begin{array}{cccc}
\rho(r) & q(r) & 0 & 0 \\
o(r) & -p_{r}(r) & 0 & 0 \\
0 & 0 & -p_{\mathrm{a}}(r) & 0 \\
0 & 0 & 0 & -p_{\mathrm{a}}(r)
\end{array}\right)
$$

The $t$, $r r$ and $\theta \theta$ components of the Einstein equation are:

$$
\begin{aligned}
-2\left(C_{r} / \sqrt{A}\right)^{\prime} / \sqrt{A}+1 / r^{2}-2 C_{r} /(r A) & \\
+C_{t}^{2} / B-C_{r}^{2} / A+2 C_{t} D_{r} / B-\Lambda & =8 \pi G \rho, \\
1 / r^{2}+C_{t}^{2} / B-C_{r}^{2} / A-2 C_{r} D_{t} / B-\Lambda & =-8 \pi G p_{r}, \\
-\left(D_{t} \sqrt{A / B}\right)^{\prime} / \sqrt{A B}-\left(C_{r} / \sqrt{A}\right)^{\prime} / \sqrt{A}-C_{r} /(r A) & \\
+C_{t} D_{r} / B-C_{r} D_{t} / B-\Lambda & =-8 \pi G p_{\mathrm{a}} .
\end{aligned}
$$

The two off-diagonal components read

$$
\begin{aligned}
-2\left(C_{t} / \sqrt{B}\right)^{\prime} / \sqrt{A}-2 C_{t} /(r \sqrt{A B})+2 C_{r} D_{r} / \sqrt{A B} & =8 \pi G o \\
-2 C_{t} D_{t} \sqrt{A / B} / B & =8 \pi G q .
\end{aligned}
$$




\subsection{Cartan's equation}

The torsion as $\mathbb{R}^{4}$-valued 2-form,

$$
T:=\mathrm{D} e=\mathrm{d} e+\omega e,
$$

has the following non-vanishing components, $T^{a}=: \frac{1}{2} T_{a^{\prime} b c} \eta^{a^{\prime} a} e^{b} e^{c}$,

$$
\begin{aligned}
T_{t t r}=\left(D_{t}-\frac{1}{2} B^{\prime} / A\right) \sqrt{A} / B, & T_{r t r}=D_{r} / \sqrt{B}, \\
T_{\theta t \theta}=T_{\varphi t \varphi}=C_{t} / \sqrt{B}, & T_{\theta r \theta}=T_{\varphi r \varphi}=\left(C_{r}-1 / r\right) / \sqrt{A} .
\end{aligned}
$$

The Cartan equation,

$$
T^{c} e^{d} \epsilon_{a b c d}=-8 \pi G s_{a b},
$$

determines the torsion in terms of its source, the half-integer spin current. This is the Lorentz-valued 3-form $s_{a b}$ i.e. the variation of the matter Lagrangian with respect to the spin connection $\omega_{a b}$. To simplify the Cartan equation, let us decompose the torsion tensor into its three irreducible parts:

$$
T_{a b c}=A_{a b c}+\eta_{a b} V_{c}-\eta_{a c} V_{b}+M_{a b c}
$$

with the completely antisymmetric part $A_{a b c}:=\frac{1}{3}\left(T_{a b c}+T_{c a b}+T_{b c a}\right)$, the vector part $V_{c}:=\frac{1}{3} T_{a b c} \eta^{a b}$, and the mixed part $M_{a b c}$ characterized by $M_{a b c}=-M_{a c b}, M_{a b c} \eta^{a b}=0$, and $M_{a b c}+M_{c a b}+M_{b c a}=0$. Likewise, we decompose the spin tensor $s_{a b c}$ defined by $* s_{a b}=: s_{a b c} e^{c}$

$$
s_{a b c}=a_{a b c}+\eta_{c a} s_{b}-\eta_{c b} s_{a}+m_{a b c},
$$

with the completely antisymmetric part $a_{a b c}:=\frac{1}{3}\left(s_{a b c}+s_{c a b}+s_{b c a}\right)$, the vector part $s_{b}:=\frac{1}{3} s_{a b c} \eta^{a c}$, and the mixed part $m_{a b c}$ characterized by $m_{a b c}=-m_{b a c}, m_{a b c} \eta^{a c}=0$, and $m_{a b c}+m_{c a b}+m_{b c a}=0$.

Then the Cartan equation reads:

$$
A_{a b c}=-8 \pi G a_{a b c}, \quad V_{a}=\frac{1}{2} 8 \pi G s_{a}, \quad M_{c a b}=-8 \pi G m_{a b c} .
$$

In the static, spherical case, we have $A_{a b c}=0$,

$$
V_{t}=\frac{1}{3}\left(2 C_{t}+D_{r}\right) / \sqrt{B}, \quad V_{r}=\frac{1}{3}\left(-\frac{1}{2} B^{\prime} / B-2 / r+2 C_{r}+D_{t} A / B\right) / \sqrt{A},
$$

and

$$
\begin{array}{cl}
M_{r t r}=\frac{2}{3}\left(-C_{t}+D_{r}\right) / \sqrt{B}, & M_{t r t}=\frac{2}{3}\left(\frac{1}{2} B^{\prime} / B-1 / r+C_{r}-D_{t} A / B\right) / \sqrt{A}, \\
M_{\theta t \theta}=M_{\varphi t \varphi}=-\frac{1}{2} M_{r t r}, & M_{\theta r \theta}=M_{\varphi r \varphi}=\frac{1}{2} M_{t r t} .
\end{array}
$$

\subsection{Second step: vacuum solution}

In vacuum, $\tau_{a b}=0$ and $s_{a b c}=0$, we retrieve the Kottler solution, equation (7). Indeed by Cartan's equation, vanishing spin current implies vanishing torsion: $C_{t}=D_{r}=0$, $C_{r}=1 / r$ and $D_{t}=\frac{1}{2} B^{\prime} / A$ and then the invariant, metric connection (19-22) reduces to the (symmetric) Christoffel symbols. 


\section{Third step: a Schwarzschild star with torsion}

Note that metric and connection in the static, spherical case are automatically invariant under space inversion. (This is not true in the homogeneous, isotropic case [4, 6].) However we do not have invariance under time reversal and to be precise we should say 'stationary' rather than 'static'.

To construct a counter example to the weak Gauß law, we do suppose invariance under time reversal. This is certainly not justified for our sun, but not unreasonable for a Schwarzschild star with constant mass density $\mathrm{d} \rho / \mathrm{d} r=0$ inside the radius $R$. The following functions are odd under time reversal and must vanish: all connection, curvature, Ricci, energy-momentum, torsion and spin tensor components with an odd number of indices equal to $t$. Consequently $C_{t}, D_{r}, q, o, s_{t}$ and $m_{r t r}$ are zero, the energy momentum tensor is symmetric and we remain with four unknown functions of $r$ in the fields: $B, A, C_{r}$ and $D_{t}$. In the sources we still have five arbitrary functions of $r$ : the mass density $\rho$ the radial and azimuthal pressure $p_{r}, p_{\mathrm{a}}$ and the spin densities $s_{r}$ and $m_{t r t}$. They define the right-hand sides of the five remaining field equations, three Einstein and two Cartan equations.

To continue, we set $D_{t}=\frac{1}{2} B^{\prime} / A$ and simplify notations $C:=C_{r}, s:=s_{r}$. Then the two Cartan equations reduce to:

$$
\frac{4}{3}(C-1 / r) / \sqrt{A}=8 \pi G s, \quad m_{t r t}=\frac{1}{2} s .
$$

Now we may introduce a Schwarzschild star by assuming that the mass density $\rho$ and the spin density $s$ are constant, i.e. $r$-independent, with an equation of state: $s=\frac{2}{3} w \rho$.

Upon eliminating $C$ via the Cartan equation, the $t t, r r$ and $\theta \theta$ components (39- 41) of Einstein's equation reduce to:

$$
\begin{aligned}
& A^{\prime} /\left(r A^{2}\right)+1 / r^{2}-1 /\left(r^{2} A\right)-16 \pi G w \rho /(r \sqrt{A})-(4 \pi G w \rho)^{2}-\Lambda=8 \pi G \rho, \\
& {[1 /(r A)+4 \pi G w \rho / \sqrt{A}] B^{\prime} / B} \\
& \quad-1 / r^{2}+1 /\left(r^{2} A\right)+8 \pi G w \rho /(r \sqrt{A})+(4 \pi G w \rho)^{2}+\Lambda=8 \pi G p_{r}, \\
& \frac{1}{2} B^{\prime \prime} /(A B)-\frac{1}{4}\left(A^{\prime} / A+B^{\prime} / B\right) B^{\prime} /(A B) \\
& -\frac{1}{2}\left(A^{\prime} / A-B^{\prime} / B\right) /(r A)+\left(\frac{1}{2} B^{\prime} / B+1 / r\right) 4 \pi G w \rho / \sqrt{A}+\Lambda=8 \pi G p_{\mathrm{a}} .
\end{aligned}
$$

As with zero torsion, the $t t$ component decouples from the other two equations and can be integrated separately. To redo the third step of section 2 for the $t t$ component with torsion, we now need two definitions of mass: an interior mass,

$$
M_{i}:=\int_{0}^{R} \rho 4 \pi \tilde{r}^{2} \mathrm{~d} \tilde{r}=\frac{4}{3} \pi R^{3} \rho, \quad \rho=\tau_{\nu=t}^{\mu=t}=\tau_{b=t}^{a=t},
$$

and an exterior mass $M_{e}$ defined by the strength of the gravitational field outside, $r \geq R$,

$$
A(r)=:\left[1-\frac{2 G M_{e}}{r}-\frac{1}{3} \Lambda r^{2}\right]^{-1} .
$$


In contrast to the torsionless case, they do not coincide:

$$
M_{e}=M_{i}\left[1+\frac{6 w}{R^{3}} \int_{0}^{R} \frac{\tilde{r} \mathrm{~d} \tilde{r}}{\sqrt{A(\tilde{r})}}+\frac{3 w^{2} G M_{i}}{2 R^{3}}\right] .
$$

Note that for sufficiently large $|w|$ the exterior mass exceeds the interior one, even for negative $w$. Note also that this mass relation depends on the interior solution $A(r)$ of the $t t$ component of the Einstein equation (55). This solution is not obvious (to us) and we will solve equation (55) numerically. As a test the numerical solution will reproduce

for $w=0$ the Schwarzschild star with cosmological constant [7, 8, 9]. This solution has $p_{r}=p_{\mathrm{a}}=: p$. Its functions,

$$
A=\frac{1}{W^{2}}, \quad B=(\alpha K+\beta W)^{2}, \quad p=\rho\left[\frac{K}{\alpha K+\beta W}-1\right],
$$

are continuous at the boundary $r=R$. The auxiliary quantities used are:

$$
\begin{aligned}
& \gamma:=\frac{1}{3}(8 \pi G \rho+\Lambda), \quad \alpha:=\frac{1}{2} 8 \pi G \rho / \gamma, \quad \beta:=\left(-\frac{1}{6} 8 \pi G \rho+\frac{1}{3} \Lambda\right) / \gamma=1-\alpha, \\
& W(r):=\sqrt{1-\gamma r^{2}}, \quad K:=W(R) .
\end{aligned}
$$

\section{Numerical solution}

Equation (55) has an integrable singularity at $r=0$ which can be avoided by redefining the dependent variable, $a(r):=r / A(r)$, yielding:

$$
1-a^{\prime}-16 \pi G w \rho \sqrt{r a}-(4 \pi G w \rho r)^{2}-\Lambda r^{2}=8 \pi G \rho r^{2} .
$$

We solve this equation by a Runge-Kutta algorithm with initial condition $a(0)=0$ for $0 \leq r \leq R$. We check that $a(r)$ remains positive for $0<r \leq R$, and that $\lim _{r \rightarrow 0} A(r)=1$ which also ensures that that $r / \sqrt{A(r)}=\sqrt{r a(r)}$ is integrable for $0 \leq r \leq R$. Then we get the masses from

$$
M_{i}=\frac{4}{3} \pi R^{3} \rho, \quad M_{e}=\left(R-\frac{1}{3} \Lambda R^{3}-a(R)\right) /(2 G) .
$$

For $w=0$ we reproduce the analytic solution $A=\left[1-\frac{1}{3}(8 \pi G \rho+\Lambda) r^{2}\right]^{-1}$ and have $M_{i}=M_{e}$ in accordance with the weak Gauß law. To obtain a ratio of $M_{e} / M_{i}=5$ for the sun, $M_{i}=M_{\odot}, R=7 \cdot 10^{8} \mathrm{~m}$, we must choose $w=3.1 \mathrm{~s}$. We get the same ratio for a cluster, $M_{i}=10^{15} M_{\odot}, R=3 \cdot 10^{23} \mathrm{~m}$, with $w=1.33 \cdot 10^{15} \mathrm{~s}$. In these two cases the positive definite contribution $\frac{3}{2} w^{2} G M_{i} / R^{3}$ to the mass ratio amounts to $6 \cdot 10^{-6}$ and 0.6 respectively. We have used the experimentally favoured value of $\Lambda=1.5 \cdot 10^{-52} \mathrm{~m}^{-2}$. Setting the cosmological constant to zero however does not change the values of $M_{e} / M_{i}$ by more than $10^{-5}$. 


\section{Conclusion}

A torsion induced failure of the weak Gauß law might be welcome with respect to some of the dark matter problems. Indeed we have already seen [4] that the Hubble diagram of super novae can be fitted by the Einstein-Cartan theory with $w=10^{17} \mathrm{~s}$ and no dark matter. This $w$-value is not far from the one found here for a spherical cluster. However they are far, far away from the naive microscopic value:

$$
w=\frac{\hbar / 2}{m_{\text {proton }} c^{2}} \sim 10^{-25} \mathrm{~s} .
$$

It would nevertheless be interesting to compute the rotation curve of a realistic galaxy and lensing in the Einstein-Cartan theory. Both are formidable theoretical challenges.

\section{References}

[1] É. Cartan, Sur les variétés à connexion affine et la théorie de la rélativité généralisée (première partie), Ann. Éc. Norm. Sup. 40 (1923) 325.

(première partie, suite), Ann. Éc. Norm. Sup. 41 (1924) 1.

(deuxième partie), Ann. Éc. Norm. Sup. 42 (1925) 17.

[2] For a review see:

F. W. Hehl, P. von der Heyde, G. D. Kerlick and J. M. Nester, General relativity with spin and torsion: Foundations and prospects, Rev. Mod. Phys. 48 (1976) 393.

[3] Three recent reviews are:

S. Capozziello, G. Lambiase and C. Stornaiolo, Geometric classification of the torsion tensor in space-time, Annalen Phys. 10 (2001) 713. |gr-qc/0101038.

I. L. Shapiro, Physical aspects of the space-time torsion, Phys. Rept. 357 (2002) 113. hep-th/0103093.

M. Blagojević and F. W. Hehl (eds.), Gauge Theories of Gravitation, a reader with commentaries (2012) Imperial College Press, London, in press.

[4] A. Tilquin and T. Schücker, Torsion, an alternative to dark matter?, arXiv:1104.0160 [astro-ph.CO], Gen. Rel. Grav. 43 (2011) 2965.

[5] M. Göckeler and T. Schücker, Differential Geometry, Gauge Theories, and Gravity (1987) Cambridge Monographs on Mathematical Physics, Cambridge University Press.

[6] T. Schücker and A. Tilquin, Torsion, an alternative to the cosmological constant?, Int. J. Mod. Phys. D 21 (2012) 1250089 [arXiv:1109.4568 [astro-ph.CO]].

[7] Z. Stuchlík, Spherically Symmetric Static Configurations of Uniform Density in Spacetimes with a Non-Zero Cosmological Constant,' Acta Phys. Slov. 50 (2000) 219, arXiv:0803.2530 [gr-qc]. 
[8] C. G. Boehmer, Eleven spherically symmetric constant density solutions with cosmological constant, arXiv:gr-qc/0312027, Gen. Rel. Grav. 36 (2004) 1039.

[9] T. Schücker, Lensing in an interior Kottler solution, arXiv:0903.2940 [astro-ph], Gen. Rel. Grav. 42 (2010) 1991 